\title{
An E-pHEMT Self-biased and Self-synchronous Class E Rectifier
}

\author{
M. N. Ruiz and J. A. García. \\ Dept. of Communications Engineering, University of Cantabria, 39005 Santander, SPAIN
}

\begin{abstract}
In this paper, the design of a self-biased and selfsynchronous class $E$ rectifier, based on an Enhancement-mode Pseudomorphic High Electron Mobility Transistor (E-pHEMT), is proposed. Characterized by a small value of the switch-mode time-constant (the on-state resistance times the output capacitance), high power efficiency figures may be obtained when forcing zero-voltage and zero-voltage-derivative switching conditions (ZVS and ZVDS). The self-synchronous operation, made possible by the device gate-to-drain coupling capacitance, leads to a compact design, while the gate-to-source Schottky junction allows self-biasing the gate terminal in order to improve the efficiency versus input power profile. Simulations, based on an extracted simplified non-linear model, are combined with measured results for implementations at $900 \mathrm{MHz}$ and $2.45 \mathrm{GHz}$. Efficiency values as high as $76 \%$ and $64 \%$ have been estimated at power levels of $-4 \mathrm{dBm}$ and $-1 \mathrm{dBm}$, respectively, with peak figures of $88 \%$ and $77 \%$.
\end{abstract}

Index Terms - Class E, E-pHEMT, nonlinear model, rectifiers, rectennas, wireless powering.

\section{INTRODUCTION}

During the last years, the interest on the design of highefficiency RF-to-DC rectifying circuits has increased significantly, conceived in this case as part of the rectifying antenna or rectenna [1], to be employed in the receiving end of a far-field wireless power transmission link or in an high frequency electromagnetic energy harvester [2]. Although usually designed over Schottky diodes, due to their benefits in terms of simplicity, compactness and cost, some efforts have also appeared in the introduction of synchronous or transistorbased rectifiers [3]. Using E-pHEMT commercial devices, high efficiency figures were reported for relatively high power levels, more typical of power beaming applications, without requiring an auxiliary bias source for the gate terminal. A low power density topology resulted, associated to the use of a complex multi-harmonic drain terminating network and the introduction of an external path for properly synchronizing the gate-to-source and drain-to-source voltage waveforms. The performance also deteriorated at lower power values, as the excitation signal amplitude was far from forcing the desired device operation as a switch.

In this paper, simple lumped element drain and gate terminating networks are employed, based the first on a selfresonant coil [4], while aimed the second to take full advantage of the device intrinsic drain-to-gate reactive signal path [5]. A gate self-biasing mechanism is also added to maximize the output conductance variation, and consequently the rectifier efficiency, along a significant input power range.

\section{E-PHEMT CHARACTERIZATION AND MODELING}

As power should be delivered to the DC load resistor, a rectifying transistor operates in the third quadrant of its $\mathrm{I} / \mathrm{V}$ characteristics when in the conduction or on-state [6]. Most available non-linear models for MESFETs and HEMTs have been conceived for their use as current-source mode amplifiers, reason why their prediction capabilities are far from being the best in the linear region and sometimes fail reproducing the inverse operation $\left(V_{d s}\right.$ and $\left.I_{d s}<0\right)$. Some alternatives were proposed nearly 15 years ago, as [7] and [8], motivated by a great interest in the design of highly linear controlled attenuators, switches and resistive mixers. The real symmetry of the device behavior suggested the use of $V_{g d}$ instead of $V_{d s}$ as a control voltage. In more recent papers, the case of [9], a slightly different strategy has been followed with very good fitting of the device global characteristics.

Based on those works, a simple non-linear model, whose equivalent circuit appears in Fig. 1, has been extracted for the selected device, the VMMK-1218, a $0.25 \mu \mathrm{m}$ gate E-pHEMT in a GaAsCap wafer-scale sub-miniature leadless package from Avago Semiconductors. While the $C_{g s}, C_{g d}$ and $C_{d s}$ capacitances have been assumed to be linear and extracted from $[\mathrm{S}]$ parameter measurements in off-state, the $I_{g s}\left(V_{g s}\right)$ and $I_{g d}\left(V_{g d}\right)$ nonlinearities have been adjusted to the widely accepted Schottky junction equation.

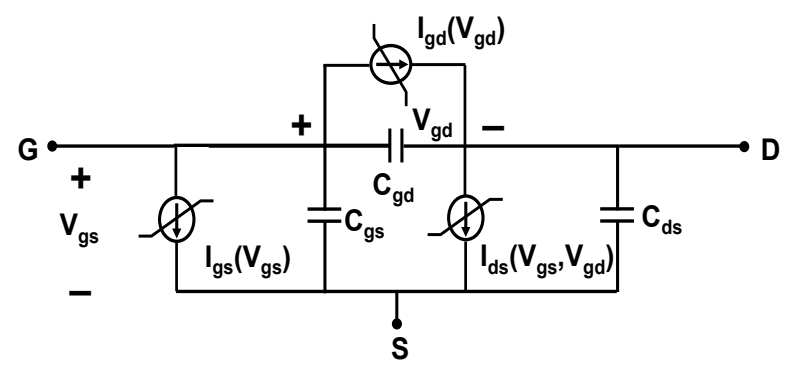

Fig. 1 Electrical equivalent circuit of the extracted model.

The $I_{d s}\left(V_{g s}, V_{g d}\right)$ main nonlinearity, modeled through eq. (1), includes continuous and continuously derivable functions for accurately describing the exponential nature of the subthreshold device behavior. Special attention was also put in the reproduction of the linear regions in normal and inverse operation (first and third quadrant).

$$
\begin{aligned}
& I d s\left(V_{g s}, V_{g d}\right)=\beta\left[1+\lambda\left(V_{g s}-V_{g d}\right)\right]\left\{\left[\alpha\left(V_{g s}-V_{t h}\right)+\right.\right. \\
& \left.\ln \left(2 \cosh \left(\alpha\left(V_{g s}-V_{t h}\right)\right)\right)\right]\left(1+\mu V_{g s}\right)-\left[\alpha\left(V_{g d}-V_{t h}\right)+\right. \\
& \left.\left.\ln \left(2 \cosh \left(\alpha\left(V_{g d}-V_{t h}\right)\right)\right)\right]\left(1+\mu V_{g d}\right)\right\}
\end{aligned}
$$


where $\beta=15 \mathrm{mS}, \alpha=7 \mathrm{~V}^{-1}, \mu=0.25 \mathrm{~V}^{-1}, V_{t h}=0.6 \mathrm{~V}$ and $\lambda$ $=0.135 \mathrm{~V}^{-1}$.

As it may be appreciated from Fig. 2, a good agreement exists between the measured and modeled characteristics, despite the relative simplicity of the adjusted equation.

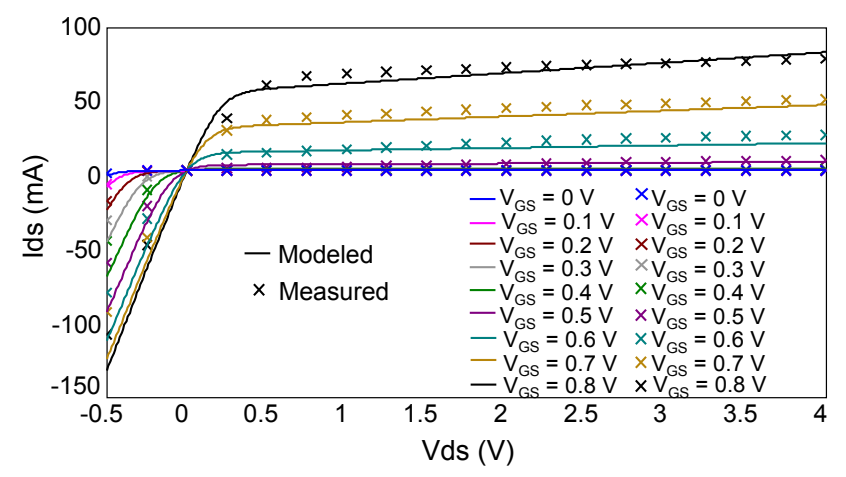

Fig. 2 Comparison of I/V measured and modeled characteristics.

\section{DRAIN AND GATE TERMINATING IMPEDANCES}

The nominal drain termination at the fundamental, theoretically derived for assuring $\mathrm{ZVS} / \mathrm{ZVDS}$ operation, $Z_{\text {opt }}(f)$ $=[0.1836+j \cdot 0.2116] /\left(\omega \cdot C_{o u t}\right)$, was estimated from the extracted device capacitances. Using the above presented model, a load-pull simulation with the VMMK-1218 operating in inverting mode and open conditions to the second and third harmonic showed that the maximum efficiency appeared very close the theoretical value, $170+\mathrm{j} \cdot 196 \Omega$.

After fixing this value at the drain terminal, load-pull simulations at gate side, following the procedure employed in [5], were carried out for different power levels and $V_{G S}$ values. The output DC resistance, $R_{D C}=380 \Omega$, was derived from the inverter simulations and approximately fitted the theoretical $R_{D C}=V_{D D} / I_{D D}=1 /\left(\pi \cdot \omega \cdot C_{o u t}\right)$ equation. As it may be appreciated from Fig. 3, for two different power levels and the $V_{G S}$ voltage where maximum efficiency was estimated (a value decreasing with the RF power), the optimum reflection coefficient at gate side was nearly the same.

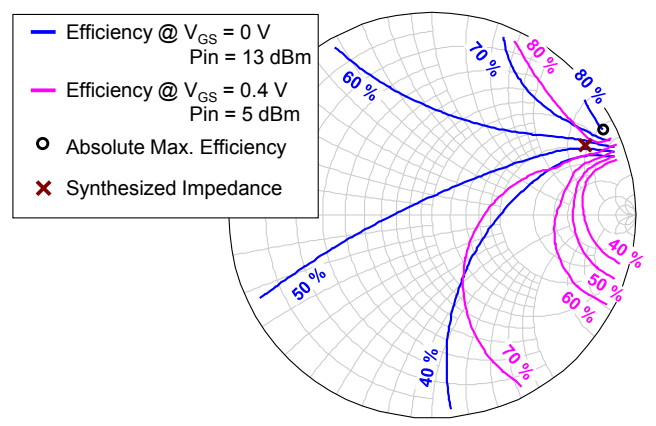

Fig. 3 Load-pull simulation results in self-synchronous mode.

Evaluating the PI reactive part of the model $\left(C_{g s}, C_{g d}\right.$ and $\left.C_{d s}\right)$ in Fig. 1 with such a termination at gate side, the drain-togate voltage transfer function was estimated to be $0.19 \cdot \exp \left(-\mathrm{j} \cdot 180^{\circ}\right)$, which means optimum self-synchronous operation occurs when the fundamental components of $V_{g s}(t)$ and $V_{d s}(t)$ are opposite phase, while their absolute values are related by $1 / G_{v}$, with $G_{v}=\left|V_{d s}(f) / V_{g s}(f)\right|$ the voltage gain (14 $\mathrm{dB}$ ) estimated from the simulations of the class $\mathrm{E}$ amplifier, its time-reversal dual.

\section{RECTIFIER TOPOLOGY AND MEASURED RESUlTS}

The proposed lumped-element circuit topology for the class E rectifier appears in Fig. 4a, together with details of the implementation in Fig. 4b. A table with the values is also provided. Ultra-miniature high current air core inductors from Coilcraft and 100A multilayer ceramic capacitors from ATC have been employed. a)

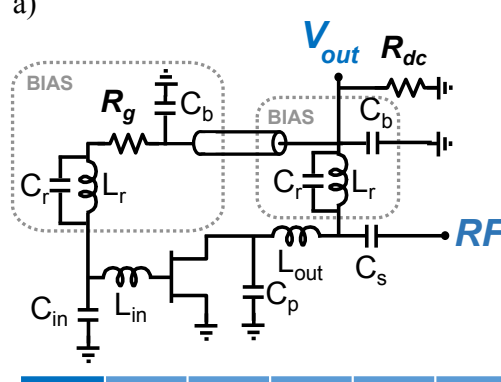

b)

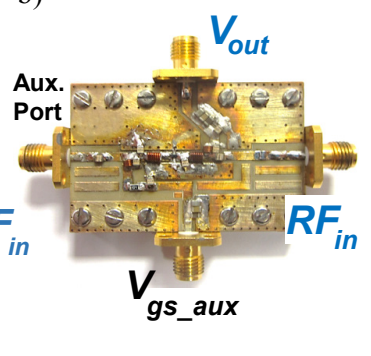

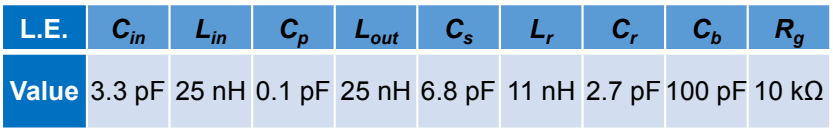

Fig 4 a) Class E rectifier schematic and b) implementation at 900 $\mathrm{MHz}$.

The $L_{\text {out }}$ coil at drain side was selected with a self-resonant frequency between the second and third harmonic [4]. The series capacitance $C_{s}$, resonating $L_{\text {out }}$, provides the required inductive termination at the fundamental, while $C_{p}$ allowed adjusting the real part of the optimum impedance. Parallel resonant circuits $\left(L_{r} C_{r}\right)$ were used instead of chokes at the gate and drain DC paths to force an open condition at the fundamental. The $C_{i n} L_{i n}$ gate network was designed for approximating the optimum gate termination. The synthesized gate impedance has been also included in Fig. 3 .

The rectifier performance was then characterized versus input power for different gate-to-source biasing voltages, as represented in Fig. 5a. The efficiency profile may peak at different power levels if such a voltage were conveniently adjusted following the represented optimum law, also derived from the load-pull simulations in section III. For very low power levels, the best conversion efficiency appears at $V_{G S}=$ $0.37 \mathrm{~V}$, relatively close to $V_{t h}$. Describing the threshold voltage the point where the device output conductance has a maximum variation with $V_{g s}$, the greatest difference between the off-state and on-state resistances may be forced at this point for small available gate driving amplitudes. When the input power level increases, the desired device performance as 
a switch is better approximated if lowering such a voltage below threshold.

The proposed self-biased topology is based on the connection of the output DC voltage port (drain side) to the gate biasing path. In this way, when increasing the input power, the rectified voltage allows increasing $V_{G S}$ up to close the threshold value (see blue trace in Fig. $5 \mathrm{~b}$, for $P_{\text {in }}<-4$ $\mathrm{dBm}$ ). As observed from Fig. 5a, the efficiency suddenly grows thanks to this self-biased effect.

Above this power value, keeping a high efficiency would demand decreasing $V_{G S}$ along the optimum path (gray dashed trace in Fig. 5b). Advantage may be taken from the small value of the rectified current appearing at gate terminal, associated to the $I_{g s}\left(V_{g s}\right)$ Schottky junction nonlinearity (see model schematic in Fig. 1). A properly dimensioned resistor, $R_{g}=10 \mathrm{k} \Omega$, introduced in the gate biasing path, allows reducing the gate-to-source DC voltage, following $V_{G S}=V_{\text {out }}$ $R_{g} \cdot I_{G S}$, close to the optimum trajectory.

As it may be appreciated in Fig. 5a, a peak value of $88 \%$ was obtained at $16 \mathrm{dBm}\left(V_{G S}=-0.36 \mathrm{~V}\right)$, while the efficiency was as high as $76 \%$ at a power value of $-4 \mathrm{dBm}\left(V_{G S}=0.31 \mathrm{~V}\right)$.

a)

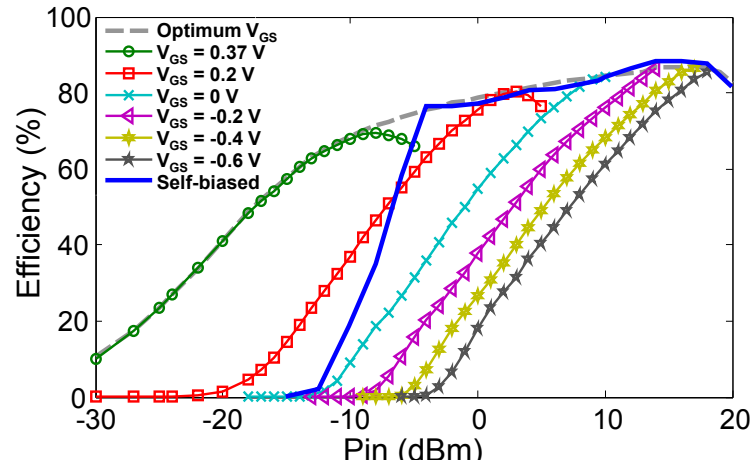

b)

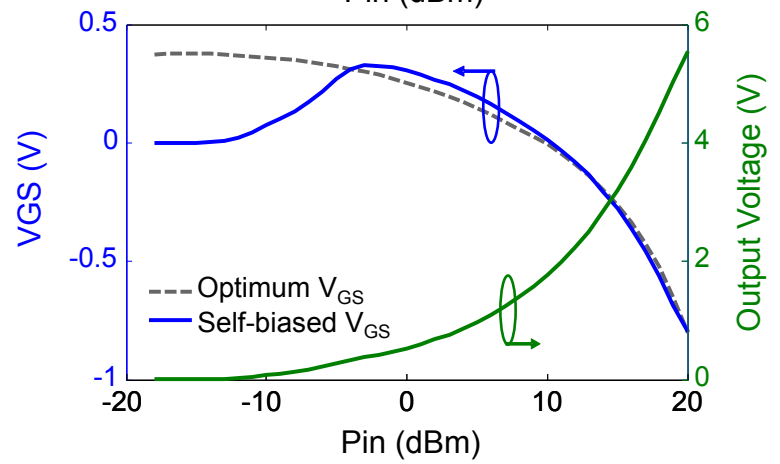

Fig. 5. Measured profiles with input power for the a) efficiency, b) the output and the $V_{G S}$ voltage.

A similar design procedure was followed for a $2.45 \mathrm{GHz}$ rectifier, based on the same VMMK-1218 device. In Fig. 6, the measured evolution of output voltage and efficiency are plotted versus input power. The estimated peak was in this case of $77 \%$ at $13 \mathrm{dBm}$, with a $64 \%$ value at $-1 \mathrm{dBm}$.

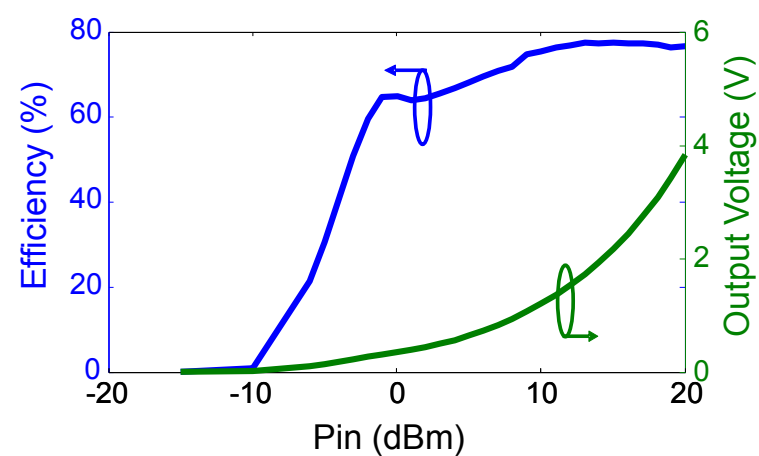

Fig. 6. Measured output voltage and efficiency profiles versus input power for a $2.45 \mathrm{GHz}$ implementation.

These results, at a higher frequency band, support the validity of the proposed design methodology, the accuracy of the extracted device nonlinear model for rectifier simulation, as well as the potential of the employed E-pHEMT technology. If interested in a broadband operation, attention should be put to the quality factor of the drain terminating network at the fundamental as well as to the evolution of the best gate side impedance condition. Employing a transistor instead of a diode, besides the flexibility associated to the auxiliary control provided by the gate voltage, the circuit may be reconfigured for operation in an inverse rectenna mode (class E oscillator) [10].

\section{CONCLUSION}

The design of a self-biased and self-synchronous class $\mathrm{E}$ rectifier, based on an Enhancement-mode Pseudomorphic High Electron Mobility Transistor (E-pHEMT), has been presented. A dedicated and simple device nonlinear model, combining linear capacitances and a symmetrical $I_{d s}\left(V_{g s}, V_{g d}\right)$ equation, has been proposed and employed for simulating the device performance in this mode of operation, overcoming the limitations associated to most widespread solutions. Combining a simple drain terminating network, based on a self-resonant coil, a self-synchronous operation and a gate self-biasing strategy, the efficiency may be kept high for a significant power range.

\section{ACKNOWLEDGEMENT}

This work was supported by MINECO through projects TEC2011-29126-C03-01, co-funded with FEDER, and Consolider CSD2008-00068. The authors wish to thank Mr. Creg Ballou, Avago Tech., by his kind assistance, as well as Mrs. Sandra Pana, by the device mounting.

\section{REFERENCES}

[1] W. C. Brown et al., U. S. Patent 3434 678, Mar. 25, 1969.

[2] Z. Popovic, E. A. Falkenstein, D. Costinett, and R. Zane, "LowPower Far-Field Wireless Powering for Wireless Sensors," Proc. IEEE, vol. 101, no. 6, pp. 1397-1409, June 2013. 
[3] M. N. Ruiz, R. Marante, and J. A. García, "A Class E Synchronous Rectifier based on an E-pHEMT Device for Wireless Powering Applications," 2012 IEEE MTT-S Int. Microwave Symp., pp. 1-3, Montreal, June 2012.

[4] R. Negra and W. Bächtold, "Lumped-Element Load-Network Design for Class-E Power Amplifiers," IEEE Trans. Microwave Theory Tech., vol. 54, no. 6, pp. 2684-2690, June 2006.

[5] M. Roberg, T. Reveyrand, I. Ramos, E. A. Falkenstein and Z. Popovic, "High-Efficiency Harmonically Terminated Diode and Transistor Rectifiers," IEEE Trans. Microwave Theory Tech., vol. 60, no. 12, pp. 4043-4053, Dec. 2012.

[6] R. W. Erickson and D. Maksimovic, Fundamentals of Power Electronics, $2^{\text {nd }}$ Ed., Springer Science, NY: 2001.

[7] J. A. García, J. C. Pedro, M. L. De la Fuente, N. B. Carvalho, A. M. Sánchez, and A. T. Puente, "Resistive FET Mixer Conversion Loss and IMD Optimization by Selective Drain Bias," IEEE Trans. Microwave Theory Tech., vol. 47, no. 12, pp. 2382-2392, Dec. 1999.

[8] K. Yhland, N. Rorsman, M. García, and H. F. Merkel, "A Symmetrical Nonlinear HFET/MESFET Model Suitable for Intermodulation Analysis of Amplifiers and Resistive Mixers," IEEE Trans. Microwave Theory Tech., vol. 48, no. 1, pp. 15-22, Jan. 2000.

[9] G. Callet, J. Faraj, O. Jardel, C. Charbonniaud, J. C. Jacquet, T. Reveyrand, E. Morvan, S. Piotrowicz, J. P. Teyssier, and R. Quéré, "A New Nonlinear HEMT Model for AlGaN/GaN Switch Applications," Int. J. Microw. Wirel. Technol., 2010, 2(3-4), pp. 283-291.

[10] M. N. Ruiz, A. González, R. Marante, and J. A. García, “A Reconfigurable Class E Oscillator/Rectifier based on an EpHEMT," INMMIC Workshop, pp.1-3, Sept. 2012. 